\section{Stability of cefiderocol against clinically significant broad-spectrum oxacillinases}

Sir,

Production of carbapenemases is a major concern in Enterobacteriaceae, Pseudomonas spp. and Acinetobacter spp. since these enzymes usually confer resistance to carbapenems. Three main $\beta$-lactamase classes group the carbapenemases, the most common being the KPC-type Ambler class A, class B (NDM-1, VIM-1, IMP-1 and SPM-1 metallo-enzymes) and class D (OXA-48-like, OXA-40, OXA-23) enzymes with extended geographic spread worldwide. The OXA-48-like $\beta$-lactamases are the most prevalent carbapenemases in Enterobacteriaceae in Europe. Carbapenemases are widespread in Acinetobacter baumannii isolates, with most multidrug-resistant strains producing OXA-40-like and OXA-23like enzymes, with OXA-23-producers being the most frequent carbapenemase-producing $A$. baumannii.

Cefiderocol is a parenteral siderophore cephalosporin also known as S-649266 that possesses a unique mechanism for penetrating efficiently into Gram-negative pathogens. It uses a 'Trojan horse' strategy by binding free iron and then actively penetrates into the bacterial cell across the outer membrane of Gram-negative bacteria owing to the bacterial iron transport system. Once across the outer membrane, the iron dissociates and the cephalosporin binds to penicillin-binding proteins (PBPs), mainly PBP3, similar to other cephalosporins, to disrupt cell wall synthesis contributing to a potent antimicrobial activity against Gram-negative bacteria [1]. Cefiderocol exhibits potent in vitro and in vivo activity against all Gram-negative bacteria, including carbapenem-resistant Enterobacteriaceae, Pseudomonas aeruginosa and A. baumannii [2]. This study evaluated the stability of cefiderocol against the most prevalent carbapenem-hydrolysing class $\mathrm{D} \beta$-lactamases (CHDLs) in Enterobacteriaceae and A. baumannii, namely OXA-48, OXA-23 and OXA-40, regardless of their bacterial hosts.

The $b l a_{\mathrm{OXA}-48}$, bla $a_{\mathrm{OXA}-23}$ and $b l a_{\mathrm{OXA}-40}$ genes were amplified by PCR and were cloned into the isopropyl $\beta$-D-1thiogalactopyranoside (IPTG)-inducible vectors pET24 (Novagen, Merck, Darmstadt, Germany) and pGEX (GE Healthcare, Switzerland) giving the pET24-OXA-48, pGEX-OXA-23 and pGEX-OXA-40 recombinant plasmids, respectively, that were subsequently transformed into Escherichia coli. Induction of gene expression was performed by adding IPTG (1 mM) to the cultures for $5 \mathrm{~h}$. Following centrifugation, the pellet of induced bacterial cells producing OXA-48 was resuspended in triethanolamine buffer $\mathrm{pH} 7.2$ $(20 \mathrm{mM})$ and was disrupted by sonication using a Vibra-Cell ${ }^{\mathrm{TM}}$ 75186 sonicator (Thermo Fisher Scientific). Following filtration using a $0.22-\mu \mathrm{m}$ nitrocellulose filter, the crude extract was loaded onto a HiTrap ${ }^{\mathrm{TM}}$ Q HP anion exchange column (GE Healthcare) using an ÄKTAprime chromatography system (GE Healthcare). Presence of the $\beta$-lactamase in the flow through fractions was monitored using nitrocefin $(200 \mu \mathrm{M})$. The positive fractions were dialysed against piperazine buffer $\mathrm{pH} 9.5(20 \mathrm{mM})$ and were loaded again onto a HiTrap ${ }^{\mathrm{TM}} \mathrm{Q}$ HP anion exchange column and were eluted using a linear gradient of $\mathrm{K}_{2} \mathrm{SO}_{4}(500 \mathrm{mM})$.

Purification of OXA-23 and OXA-40 $\beta$-lactamases followed the same process except that resuspension of the pellets was done in $10 \mathrm{mM}$ sodium phosphate, $140 \mathrm{mM} \mathrm{NaCl}$ and $2.7 \mathrm{mM} \mathrm{KCl}(\mathrm{pH}$ 7.4) and elution was done using glutathione buffer (50 mM Tris$\mathrm{HCl}, 10 \mathrm{mM}$ glutathione, $\mathrm{pH}$ 8). All fractions containing purified $\beta$-lactamases were dialysed against sodium phosphate buffer $\mathrm{pH}$ $7.0(0.1 \mathrm{M})$ in order to perform kinetic measurements.

Purified $\beta$-lactamases were used for kinetic measurements performed at $30^{\circ} \mathrm{C}$ in sodium phosphate buffer pH $7.0(0.1 \mathrm{M})$. Kinetic parameters showed a significant hydrolysis rate of penicillins and carbapenems by the OXA-48, OXA-23 and OXA-40 purified enzymes, respectively, which is consistent with previous studies. Noteworthy, no enzymatic activity was detected for cefiderocol, mirroring the susceptibility test results (Table 1).

Minimum inhibitory concentrations (MICs) were determined by the broth microdilution method in iron-depleted and calciumadjusted Muller-Hinton broth medium (Bio-Rad). MICs showed that all CHDL-producing E. coli clones exhibited a high level of resistance to aminopenicillins and carboxypenicillins and intermediate resistance to imipenem (MICs of 1,4 and $8 \mathrm{mg} / \mathrm{L}$ for OXA48, OXA-40 and OXA-23, respectively, compared with $0.06 \mathrm{mg} / \mathrm{L}$ for the $E$. coli recipient), but remained fully susceptible to cefiderocol with no change in the MICs (respective values being all at $0.03 \mathrm{mg} / \mathrm{L}$ ).

Altogether, these findings highlight the stability of cefiderocol toward the hydrolytic capacity of CHDLs. Cefiderocol is a modified cephalosporin molecule with an attached catechol moiety on the 3-position side chain that binds to ferric iron [1]. It was previously shown that CHDLs do not hydrolyse broad-spectrum cephalosporins [2]. The current data thus confirm that cefiderocol act as a potent cephalosporin, even in the presence of CHDLs.

Previous studies showed that cefiderocol is active against a wide range of carbapenemase-producers, including those belonging to classes A and B [3,4]. Very recently, a study described the stability of cefiderocol against AmpC-producing Gram-negative isolates [5]. Here we demonstrated that the cefiderocol molecule is also stable against CHDL-producers, confirming that this molecule represents an interesting option for treating infections caused by carbapenemase-producing Gram-negative bacteria. 
Table 1

Kinetic parameters of the OXA-48, OXA-40 and OXA-23 carbapenemases.

\begin{tabular}{|c|c|c|c|c|c|c|c|c|c|}
\hline \multirow[t]{2}{*}{$\beta$-Lactam } & \multicolumn{3}{|l|}{ OXA-48 } & \multicolumn{3}{|l|}{ OXA-40 } & \multicolumn{3}{|l|}{ OXA-23 } \\
\hline & $K_{\mathrm{m}}(\mu \mathrm{M})$ & $k_{\text {cat }}\left(\mathrm{s}^{-1}\right)$ & $k_{\text {cat }} / K_{\mathrm{m}}\left(\mathrm{mM}^{-1} / \mathrm{s}^{-1}\right)$ & $K_{\mathrm{m}}(\mu \mathrm{M})$ & $k_{\text {cat }}\left(\mathrm{s}^{-1}\right)$ & $k_{\text {cat }} / K_{\mathrm{m}}\left(\mathrm{mM}^{-1} / \mathrm{s}^{-1}\right)$ & $K_{\mathrm{m}}(\mu \mathrm{M})$ & $k_{\text {cat }}\left(\mathrm{s}^{-1}\right)$ & $k_{\text {cat }} / K_{\mathrm{m}}\left(\mathrm{mM}^{-1} / \mathrm{s}^{-1}\right)$ \\
\hline Benzylpenicillin & 200 & 1750 & 8750 & 20 & 210 & 10500 & 60 & 40 & 670 \\
\hline Ampicillin & 1100 & 370 & 340 & 150 & 1.5 & 10 & nd & $<0.1$ & nd \\
\hline Ticarcillin & 90 & 70 & 780 & 70 & 4 & 50 & 70 & 7 & 100 \\
\hline Imipenem & 15 & 1 & 70 & 50 & 3.5 & 70 & 80 & 0.5 & 6 \\
\hline Cefiderocol & ND & $<0.1$ & ND & ND & $<0.1$ & ND & ND & $<0.1$ & ND \\
\hline
\end{tabular}

nd, not determined due to instability of the hydrolysis complex; ND, not determinable due to the absence of detectable hydrolytic activity.

\section{Funding}

This work was partially supported by SHIONOGI @ CO., LTD., Osaka, JAPAN. It was also funded by the University of Fribourg (Fribourg, Switzerland) and the Swiss National Science Foundation [project FNS-31003A_163432].

\section{Competing interests}

None declared

\section{Ethical approval}

Not required.

\section{References}

[1] Kohira N, West J, Ito A, Ito-Horiyama $T$, Nakamura $R$, Sato $T$, et al. In vitro antimicrobial activity of a siderophore cephalosporin, S-649266, against Enterobacteriaceae clinical isolates, including carbapenem-resistant strains. Antimicrob Agents Chemother 2015;60:729-34.

[2] Dobias J, Dénervaud-Tendon V, Poirel L, Nordmann P. Activity of the novel siderophore cephalosporin cefiderocol against multidrug-resistant Gram-negative pathogens. Eur J Clin Microbiol Infect Dis 2017;36:2319-27.

[3] Falagas ME, Skalidis T, Vardakas KZ, Legakis NJHellenic Cefiderocol Study Group. Activity of cefiderocol (S-649266) against carbapenem-resistant Gram-negative bacteria collected from inpatients in Greek hospitals. J Antimicrob Chemother 2017;72:1704-8

[4] Hackel MA, Tsuji M, Yamano Y, Echols R, Karlowsky JA, Sahm DF. In vitro activity of the siderophore cephalosporin, cefiderocol, against a recent collection of clinically relevant Gram-negative bacilli from North America and Europe, including carbapenem-nonsusceptible isolates (SIDERO-WT-2014 Study). Antimicrob Agents Chemother 2017;61 pii: e00093-17.

[5] Ito A, Nishikawa T, Ota M, Ito-Horiyama T, Ishibashi N, Sato T, et al. Stability and low induction propensity of cefiderocol against chromosomal AmpC $\beta$-lactamases of Pseudomonas aeruginosa and Enterobacter cloacae. J Antimicrob Chemother 2018;73:3049-52. doi:10.1093/jac/dky317.
Laurent Poirel $^{*}$

Medical and Molecular Microbiology Unit, Faculty of Science and Medicine, University of Fribourg, Fribourg, Switzerland INSERM European Unit (IAME, France), University of Fribourg, Fribourg, Switzerland Swiss National Reference Center for Emerging Antibiotic Resistance (NARA), University of Fribourg, Fribourg, Switzerland

Nicolas Kieffer Medical and Molecular Microbiology Unit, Faculty of Science and Medicine, University of Fribourg, Fribourg, Switzerland INSERM European Unit (IAME, France), University of Fribourg, Fribourg, Switzerland

Patrice Nordmann

Medical and Molecular Microbiology Unit, Faculty of Science and Medicine, University of Fribourg, Fribourg, Switzerland INSERM European Unit (IAME, France), University of Fribourg, Fribourg, Switzerland Swiss National Reference Center for Emerging Antibiotic Resistance (NARA), University of Fribourg, Fribourg, Switzerland Institute for Microbiology, University of Lausanne and University Hospital Centre, Lausanne, Switzerland

*Corresponding author. Present address: Medical and Molecular Microbiology Unit, Faculty of Science and Medicine, University of Fribourg, Chemin du Musée 18, CH-1700 Fribourg, Switzerland. E-mail address: laurent.poirel@unifr.ch (L. Poirel) 